neofilolog

Czasopismo Polskiego Towarzystwa Neofilologicznego

ISSN 1429-2173, elSSN 2545-3971, 2020, NR 54/1, 155-169

http://dx.doi.org/10.14746/n.2020.54.1.9

http://poltowneo.org/

Halina Widła

Uniwersytet Śląski

https://orcid.org/0000-0001-7384-5897

halina.widla@us.edu.pl

\title{
ÉVALUATION FORM ATIVE PAR LE BIAIS DE PRATIQUES « LERNEN DURCH LEHREN » DESTINÉES AUX ÉTUDIANTS DE NIVEAU AVANCÉ
}

\section{Formative evaluation using Lernen durch Lehren practices for advanced students}

This article illustrates ways of assessing students' work in the M ultimedia master's seminar and compares the benefits of formative and summative evaluations in this context. During the Multimedia seminar, prospective foreign language teachers and translators create their own multimedia resources, which fill a gap in teaching materials on offer. This allows the students to become specialists in particular areas. This article illustrates ways of assessing students' work in the Multimedia master's seminar and compares the benefits of formative and summative evaluations in this context. Students appreciated the importance of formative assessment, recognizing its advantage over summative evaluation. Systematic semi-autonomous work subjected to continual verification proved to be key to the achievement of the desired skills. The results also demonstrated how knowledge of learners' first and second languages is advantageous, as it allows teachers to more accurately identify and evaluate the difficulties learners face during the acquisition of a third language. These unusual forms of learning by teaching require considerable time and sufficient motivation but notably improve the quality of the learning process.

Keywords: Formative evaluation, learning by teaching, e-learning

Słowa kluczowe: Ocenianie kształtujące, uczenie się przez nauczanie, nauczanie na odległość 


\section{Introduction}

L'objectif de cet article est de montrer l'une des opportunités offertes par les nouvelles technologies de l'information et de la communication dans le cadre de l'évaluation et de l'auto-évaluation des compétences linguistiques des étudiants. Cette étude porte sur l'application d'une variante de la méthode LDL $^{1}$ auprès d'étudiants dans le cadre d'un séminaire de master. Le concept éducatif $d$ ' « apprendre en enseignant », populaire pendant des siècles en Inde, s'est répandu par la suite dans l'Europe médiévale avant de céder la place à une école où la connaissance est transmise par le maître. Bien qu'une telle approche soit recommandée par certains pédagogues, le recours à l'expérience de la « classe mutuelle » est encore sporadique. À partir de 1980, le professeur de français Jean-Pol M artin, qui est parvenu à appliquer un tel système à l'Université de Bavière, devient un propagateur actif de cette méthode de travail auprès des étudiants ; une solution suffisamment fréquente pour qu'on y porte attention. Son approche naît suite à l'analyse critique qu'il fait des méthodes traditionnelles, qu'il décrit de la manière suivante:

Les méthodes pratiquées actuellement présentent, à cet égard aussi, de graves déficits. S'adressant en premier lieu à la mémoire, les techniques traditionnelles imposent une attitude réceptive à l'apprenant et laissent peu de place au développement d'activités individualisées et autonomes ; il en est de même des approches behavioristes qui, de plus, utilisent des pratiques répétitives peu propices aux processus intellectuels complexes et à la créativité. Ces méthodes ne tenant pas compte de besoins fondamentaux des élèves, ceux-ci réagissent dans les deux cas par des comportements à la fois passifs et agressifs. Quant aux approches communicatives, qui privilégient la motivation de l'apprenant, elles ont l'inconvénient de faire supporter par le professeur tout le poids de l'effort didactique. En effet, comme il s'agit moins de faire assimiler par les élèves les contenus du manuel que de les inciter à s'exprimer dans un contexte authentique, l'enseignant doit constamment faire preuve d'imagination pour créer, au-delà des situations fournies par le livre, des occasions de parole motivantes. (Martin, 1989 : 51)

\section{Conception du séminaire Multimedia}

Le séminaire prépare les étudiants à rédiger un mémoire de master composé d'une partie théorique et d'une partie pratique. La partie théorique couvre la réflexion autour de la problématique de l'enseignement et de l'apprentissage

${ }^{1}$ de l'allemand Lernen durch Lehren (apprendre en enseignant) 
Évaluation formative par le biais de pratiques « lernen durch lehren »...

des langues de spécialité. Pour la partie pratique, et ce dans une perspective pédagogique, l'étudiant doit préparer et proposer des cours en ligne sur le sujet de son choix. Ce cours, par le biais de la problématique abordée, doit permettre une bonne immersion linguistique. Puisque, en principe, les sujets choisis par les étudiants touchent des contenus non linguistiques, la majorité des cours s'inscrivent aussi dans les pratiques CLIL/EM ILE- Enseignement de M atières par Intégration d'une Langue Étrangère (Coyle, Hood, Marsh : 2010).

\section{Déroulement des travaux répartis en quatre semestres d'études}

Au cours du premier semestre, chaque étudiant se familiarise avec les méthodes et techniques d'appropriation des langues spécialisées. Pour ce faire, il consacre beaucoup de temps à la recherche documentaire. Après avoir sélectionné les textes et avoir évalué leur pertinence et qualité, l'étudiant concrétise la thématique qu'il va aborder. Ensuite, il précise le sujet du cours en ligne qu'il va mettre en place sur la plateforme d'enseignement à distance. L'idée est d'y mettre en pratique toutes les solutions théoriques décrites dans la première partie du mémoire.

\begin{tabular}{|c|c|c|c|c|c|c|c|c|c|c|c|}
\hline \begin{tabular}{l|l|} 
Sujet & Année \\
\end{tabular} & 2009 & 2010 & 2011 & 2012 & 2013 & 2014 & 2015 & 2016 & 2017 & 2018 & Total \\
\hline littérature & 4 & 3 & & 1 & 1 & 2 & & 3 & & 2 & 14 \\
\hline linguistique & 3 & 3 & & 1 & 2 & 1 & & 1 & 2 & 2 & 15 \\
\hline didactique & & 2 & 1 & & & 4 & & & & 1 & 8 \\
\hline sublangage & & & & & 1 & 1 & & 1 & 1 & 1 & 5 \\
\hline culture/civilisation & 2 & 3 & 8 & 1 & 1 & & & & & & 15 \\
\hline écologie & & & & 1 & 1 & & & & 1 & & 3 \\
\hline géographie & & & & 1 & 1 & & 1 & 1 & & & 4 \\
\hline industrie & & & & & 1 & 1 & & 1 & 2 & 1 & 6 \\
\hline sport & & & & & & & & & 2 & 1 & 3 \\
\hline musique & & & & & & 1 & & 1 & 1 & 1 & 4 \\
\hline technologies & & & & & & & & & 1 & 1 & 2 \\
\hline Total & 9 & 11 & 9 & 5 & 8 & 10 & 1 & 8 & 10 & 10 & 81 \\
\hline
\end{tabular}

Tab. 1 : Répartition desmémoires selon les années de fin d'études et la problématique étudiée.

La liberté de choisir le sujet de son propre cours en ligne vise à combler les lacunes linguistiques ressenties par les étudiants. Ainsi, ces deux années de travail leur offrent la possibilité d'améliorer leurs compétences linguistiques ou même de devenir experts dans un domaine auparavant inexploité. Cette perspective doit créer une certaine dynamique chez les étudiants. Les participants au séminaire avouent certaines lacunes linguistiques qui freinent leur maîtrise d'une grande quantité de sujets. De la terminologie générale liée à l'histoire, à la culture, la géographie et le tourisme, le droit, le travail, Internet, 
l'enseignement des langues, l'écologie, le sport, la musique, la gastronomie, les produits de beauté, à celle permettant de maîtriser des sujets plus spécialisés comme celui de l'automobile, de l'énergie, de la joaillerie, de la pharmacie, des catastrophes naturelles, de la bande dessinée et des sous-langues, pour ne citer que quelques exemples, les besoins terminologiques sont nombreux au sein d'une même spécialité philologique.

Le second semestre est consacré à la rédaction des chapitres théoriques. Sur cette base, en vue de la mise en pratique de la théorie, ils élaborent les plans détaillés des cours, destinés à des utilisateurs du réseau intéressés par l'étude du sujet proposé dans une langue étrangère. Souvent, les auteurs adressent leurs cours à de futurs employés du secteur culturel, d'établissements scolaires ou d'entreprises diverses.

Pendant le troisième semestre, en travaillant en ligne sur leurs propres modules thématiques, les étudiants utilisent pratiquement toutes les fonctionnalités de la plateforme, maîtrisées au cours du premier semestre: enregistrements audios et vidéos, fichiers dans tous les formats avec des contenus qui deviennent la base de leçons, glossaires, exercices de toutes sortes. En pratique, tout en travaillant sur le projet, l'étudiant maîtrise le sujet, le vocabulaire et les phrases usuelles dans le contexte culturel approprié de la langue étrangère choisie.

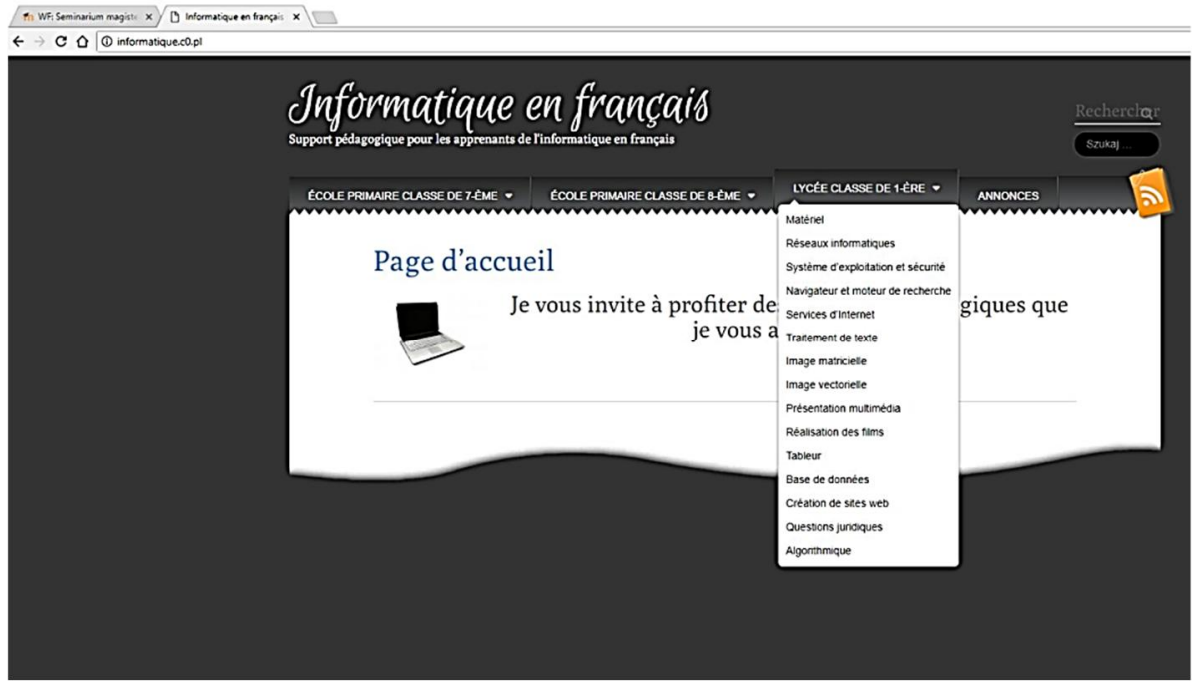

Fig. 1 : Page d'accueil du cours « Informatique en français » destiné aux collégiens et lycéens de classes bilingues, conforme au programme d'enseignement en Pologne. Auteure : Grażyna Tkocz. 
Évaluation formative par le biais de pratiques « lernen durch lehren »...

Finalement, le dernier semestre est consacré à la rédaction de la deuxième partie du mémoire, c'est-à-dire à la description des cours en ligne (accessible au jury du mémoire) sous l'angle de ses objectifs, du public visé, de la thématique, du contenu linguistique et des solutions didactiques adoptées.

\section{Exemples de projets}

Comme nous l'avons déjà souligné, les parties théoriques des mémoires abordent le sujet de l'enseignement des langues de spécialité. En expliquant de quelle façon ce type de cours diffère de l'enseignement des langues générales, les auteurs cherchent des solutions didactiques efficaces. Quant à la partie analytique où ces solutions mises en place sont décrites, les élèves du séminaire ont élaboré 81 cours de spécialité en ligne. À cela s'ajoutent encore 10 cours en préparation. Chaque cursus est divisé en modules thématiques accompagnés de différentes activités et exercices, destiné à un groupe ciblé. Un sujet exige au moins 60 heures de travail en ligne. Nous en donnons quelques exemples ci-dessous, en nous limitant à quatre projets caractéristiques.

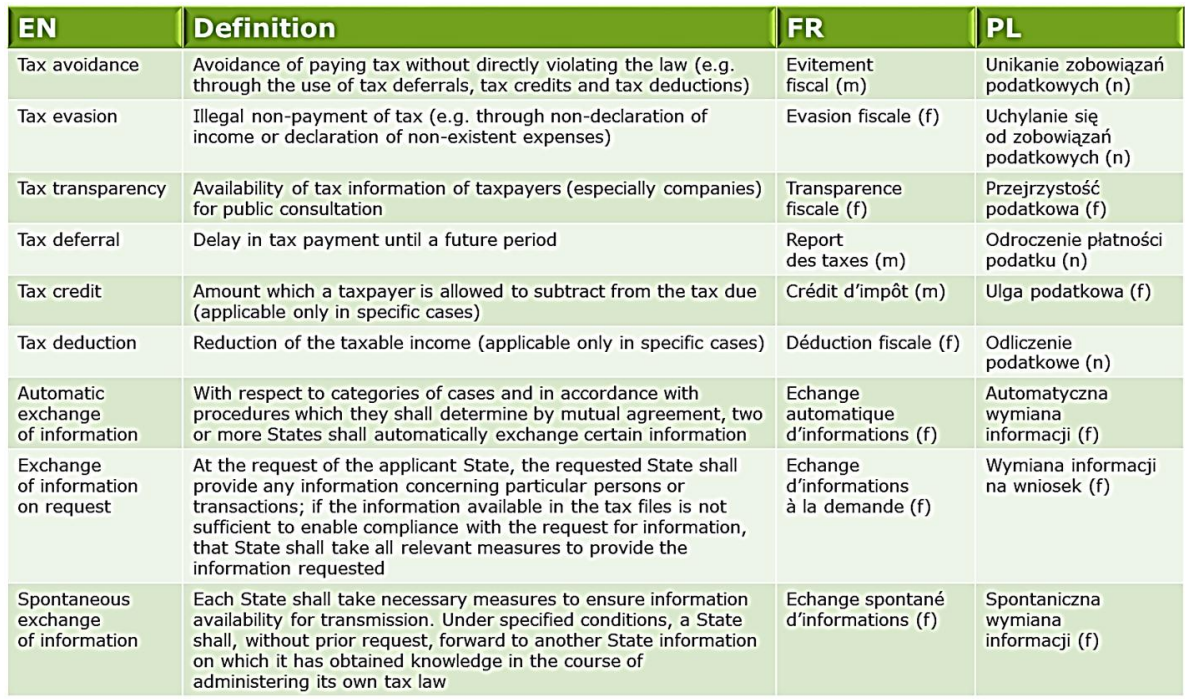

Fig. 2 : Capture d'écran provenant du cours élaboré par Magda Kurylak : «La langue internationale du droit fiscal ».

L'auteure du projet réalisé dans le cadre du mémoire « L'enseignement de la langue de spécialité fondé sur le contenu - illustré par le cours en ligne "La langue internationale du droit fiscal" », Magda Kurylak, s'est concentrée sur la langue caractéristique du droit fiscal. Elle considère celle-ci « comme étant une 
matière étroite de la langue juridique, rarement incluse dans les cours de langue variés » (Kurylak : 2018). L'étudiante a adressé son cours aux adultes dont le niveau de langue anglaise atteint au moins le niveau B2 et qui souhaitent travailler dans le domaine du droit fiscal international. Elle analyse les méthodes de transmission et d'acquisition des connaissances, en expliquant leur impact sur l'amélioration des compétences dans l'apprentissage d'une langue de spécialité.

Le mémoire de master d'Anne-Sophie Basta-Szczygieł intitulé « Maîtrise de la langue de spécialité en joaillerie - cours de français en ligne pour les traducteurs » a été divisé en deux parties. Dans la partie théorique, l'auteure explique la place de la langue de spécialité dans la terminologie. Elle présente également les bases de la phraséologie dans le contexte des langues de spécialité. Ensuite, l'auteure met l'accent sur les différentes règles « que le traducteur doit suivre pour être efficace et cohérent lors du processus de traduction » (Basta-Szczygieł, 2017). La deuxième partie propose des solutions didactiques - et les justifie - dans la création d'une aide pour le traducteur désirant approfondir ses connaissances dans le domaine de la joaillerie. Le cours, qui comporte un glossaire franco-polonais des bijoux, recense également un large éventail d'expressions utiles à un bijoutier.
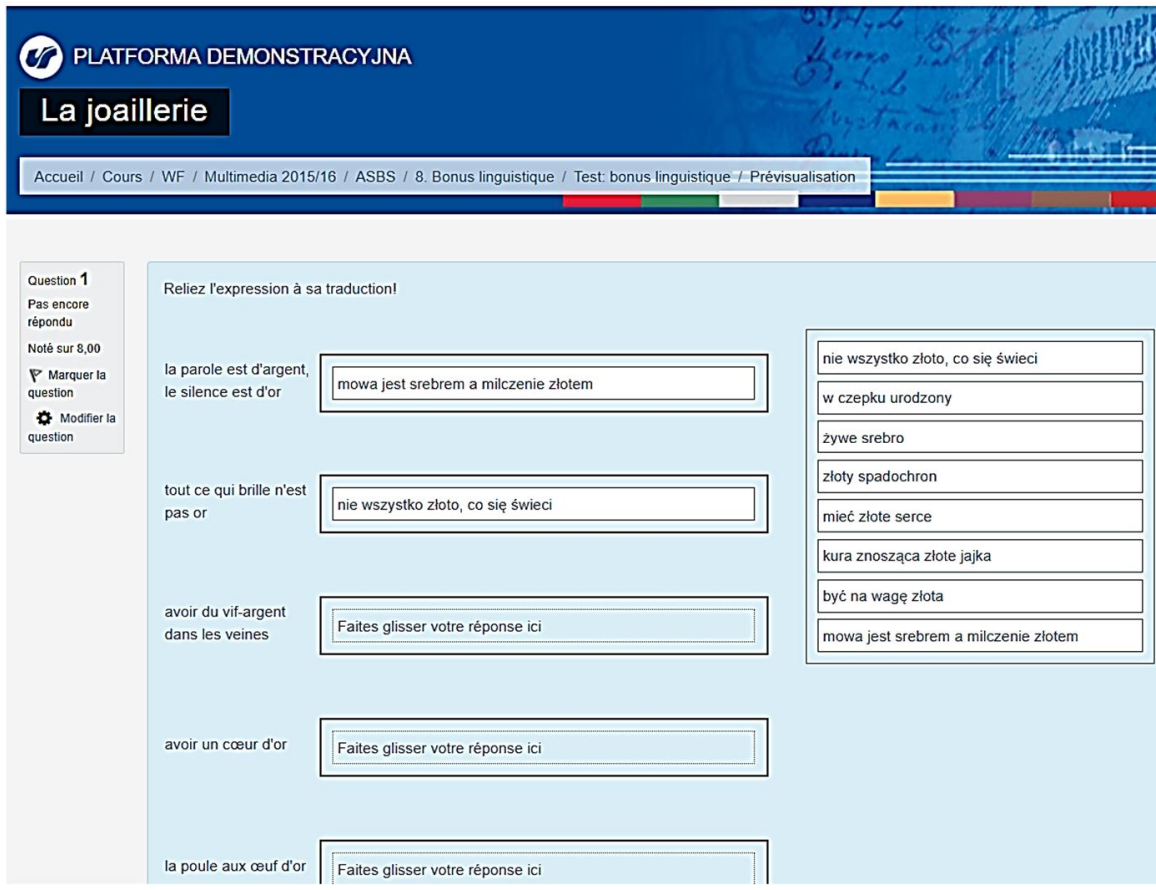

Fig. 3 : Capture d'écran provenant du cours élaboré par Anne-Sophie Basta-Szczygieł : «M aîtrise de la langue de spécialité en joaillerie - cours de français en ligne pour les traducteurs ». 
Évaluation formative par le biais de pratiques « lernen durch lehren »...

Le mémoire de master de Natalia Piwoń (2018) intitulé « Enseignement en ligne de l'anglais répondant à des objectifs spécifiques (ESP) - la communication commerciale - exemple d'un cours réalisé sur la plateforme Moodle » traite de l'importance de la communication dans le milieu commercial. La partie théorique fournit des informations sur le processus de communication. Elle explicite les différents moyens de communication, les différents aspects que ce processus peut prendre. Ce travail aborde aussi le sujet de la langue de spécialité ainsi que celui de l'apprentissage de l'anglais portant sur des objectifs spécifiques. La partie analytique présente le cours « La communication commerciale », préparé sur la plateforme Moodle, destiné aux polonophones qui maîtrisent déjà le français à un niveau avancé et possèdent au minimum le niveau d'anglais intermédiaire, ainsi qu'aux personnes qui utilisent l'anglais dans leur travail, mais constatent un manque de vocabulaire de spécialité. L'auteure a divisé son cours en sept parties thématiques : les types d'entreprises, le marketing, la vente, les ressources humaines, la gestion, le financement d'entreprise et le jargon d'entreprise.

\section{Natalia Piwoń}

Home / Courses / WF / Multimedia 2016/17 / NP / " Corporate Jargon / CORPORATE JARGON / Preview

CORPORATE JARGON O

Preview Edi Reports Grade essays

The meaning of corporate jargon

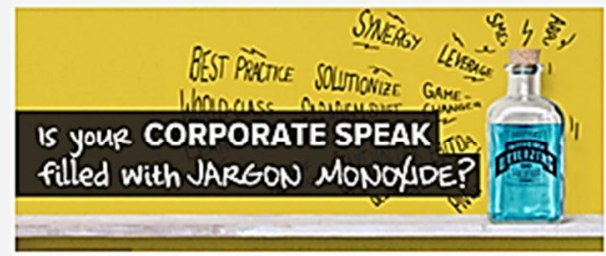

If you want to add value to your tell-mode paradigm in the competitive modern workplace, you need to keep up to date with the unhappy and stressed, and cause everyone to feel as though they spend their days in a nightmare of corporate newspeak, wher appears to say

The problem is that while everyone knows this, everyone is also well aware that they don't want to be the one person who is ridic being the lone voice of reason is no good to anyone. So if you can't beat them, join them, with this selection of the hippest, most

sourse

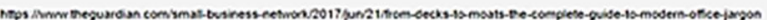

mase:

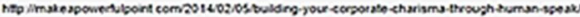

Fig. 4 : Capture d'écran provenant du cours élaboré par Natalia Piwoń : «Corporate jargon». 
Le mémoire de master de Magdalena Majcher « Idioms as a linguistictool to express feelings: idiomatic expressions in English and French - example of an online course on the M oodle platform »attire l'attention du lecteur sur la construction du sens dans certains verbes anglais à particule, les plus fréquemment utilisés pour exprimer des sentiments. La liste choisie ne se veut pas exhaustive, mais représentative. Le cours en ligne de $M$ agdalena $M$ ajcher, conçu pour les apprenants polonais en anglais aux niveaux intermédiaires et avancés, avec au moins une connaissance intermédiaire du français, a pour but de « sensibiliser les étudiants à la nature métaphorique du langage et démontrer de quelle manière les verbes à particule anglais reflètent les métaphores conceptuelles présentes dans nos esprits » (Majcher, 2018).

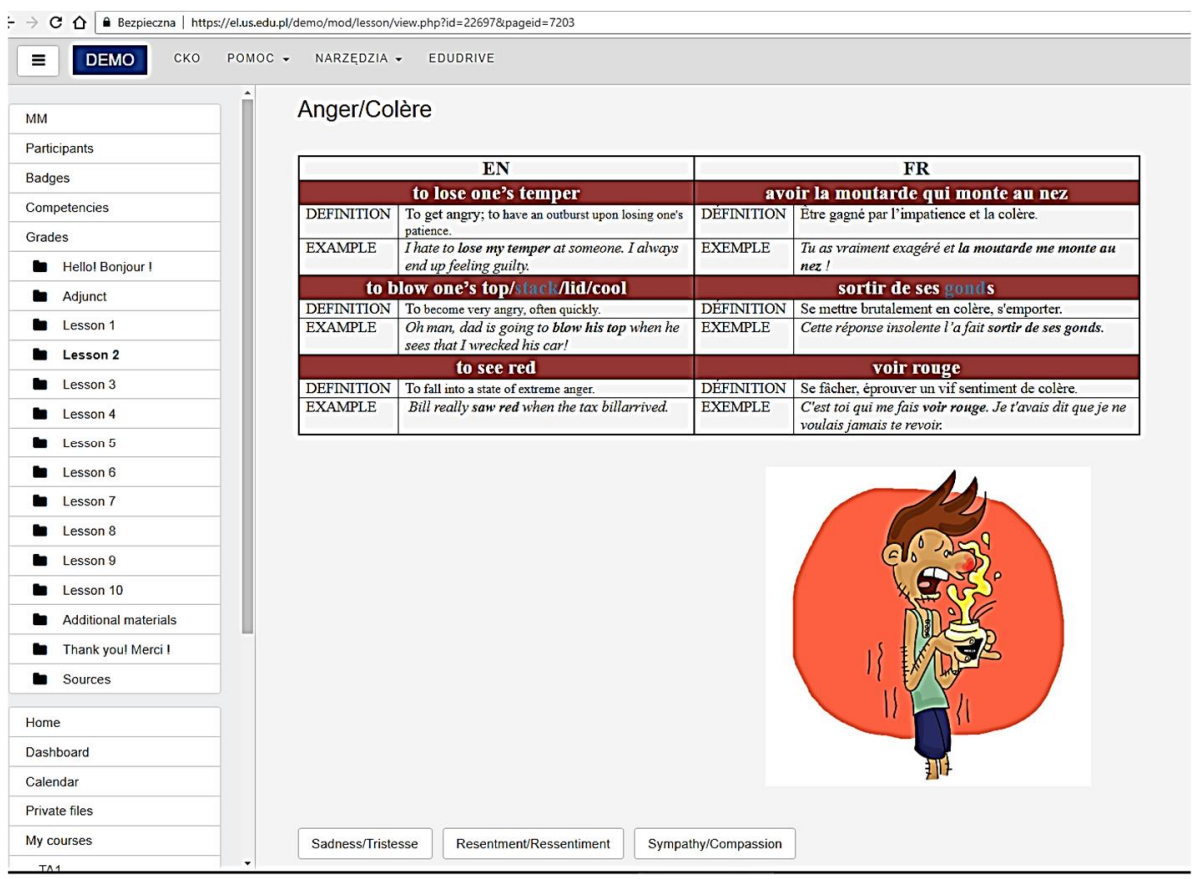

Fig. 5 : Capture d'écran provenant du cours élaboré par M agdalena M ajcher «Idioms as a linguistic tool to express feelings »

\section{Principes d'évaluation}

En ce qui concerne l'évaluation sommative des étudiants de master, les évaluateurs se concentrent sur la qualité des mémoires et soutenances, constituant pour chacun d'entre eux un quart de la note finale du diplôme. La deuxième moitié - la moyenne d'études - est calculée par le bureau du doyen. 
Ceci pris en considération, l'étudiant part souvent du principe que l'objectif primordial de sa participation au séminaire est la note finale attribuée par un jury de soutenance. Par conséquent, le problème auquel se heurtent souvent les directeurs de mémoires est celui du délai de réal isation des parties successives de mémoires. La question se pose donc de savoir comment convaincre les étudiants des bénéfices du travail dans un contexte formatif avant de se soumettre à une évaluation sommative.

La mise en place d'un séminaire tel que celui décrit dans cette étude doit débuter par une description précise des différentes étapes et intérêts de l'évaluation formative :

- connaissance détaillée des programmes prévus pendant les quatre semestres de travail

- indication des étapes à venir des processus d'apprentissage au cours des semestres consécutifs

- précision de l'objectif d'amélioration des apprentissages dans un domaine choisi

- élaboration des critères utilisés pour évaluer le travail des étudiants mettant l'accent sur la progression vers la maîtrise des compétences linguistiques plutôt que sur la validation de tâches particulières

De plus, le climat de confiance et de motivation mutuelle instauré par le responsable de mémoire doit encourager les étudiants à échanger les résultats partiels de leurs projets et à travailler encore plus intensément. Ce travail se combine évidemment aux exigences des évaluations sommatives qui complètent le but de travaux en tant qu'acquis naturellement prévisibles.

\section{6. Évaluation formative}

En réalisant leurs propres cours d'autoapprentissage de langue pendant quatre semestres, les étudiants travaillent en semi-autonomie. Le résultat de leur travail est double : ils approfondissent leurs connaissances de la problématique choisie, devenant ainsi experts dans des domaines encore peu explorés durant leurs études, tandis que leurs progrès linguistiques dans ce domaine augmentent visiblement. De plus, ils améliorent pendant leurs études leurs compétences informatiques et didactiques. Selon le programme, l'étudiant doit être capable de préparer et de mettre à disposition sur Internet des cours, du matériel d'information dans une langue étrangère, mais surtout de préparer et de diriger des cours de langues étrangères en ligne. Lors de la construction formelle des cours sur Internet, il fait généralement preuve d'une grande créativité. 
Conformément aux objectifs fixés pour les quatre semestres, exposés dans le syllabus du séminaire cité ci-dessus, un plan détaillé et individuel de progression est communément élaboré avec chaque participant. Ce plan explicite et décrit les objectifs et contenus de chaque séance de cours et fournit le calendrier détaillé des travaux à remettre. Ainsi, les problèmes théoriques exposés dans les chapitres successifs rendus au directeur de mémoire sont présentés et discutés dans les groupes. La présentation des cours programmés en ligne se fait également par étapes afin de pouvoir mettre en place toutes les corrections ou modifications résultant du brainstorming réalisé par les membres du séminaire.

La nécessité de préparer des rapports partiels, exposés par les étudiants devant le groupe et soumis à l'évaluation des progrès, exige un travail systématique. Ces interventions concernent:

- la présentation systématique des problèmes théoriques, suivie d'une suite de questions et/ou observations de la part des participants. Le séminaire se transforme alors en atelier de partage de connaissances. Dans ce cas-là, c'est l'efficacité de la transmission, la cohérence du discours et le niveau de langue qui sont analysés.

- la présentation des étapes successives des supports didactiques mis en ligne. Ici le groupe se familiarise avec un sujet particulier « creusé à fond » par l'auteur et juge la pertinence des solutions choisies sous l'angle du contenu théorique ainsi que l'imagination pédagogique numérique de l'auteur. La sélection de ressources attrayantes, le choix des fonctionnalités offertes par une plateforme ou un mobile, la conception d'exercices interactifs, l'invention d'activités ludiques, l'adéquation de tous les outils à la quantité du matériel exercé, à l'ordre de sa présentation et au niveau pertinent de destinataire sont ainsi analysés. Cet échange d'idées est jugé comme particulièrement utile et enrichissant, car il n'est pas aisé de répondre aux attentes des lecteurs qui apprennent en autodidactes.

\section{Opportunités et défis du trilinguisme}

Les étudiants participant au séminaire sont bilingues (polonais ayant appris le français) ou trilingues (polonais ayant appris l'anglais L2 et le français L3). Le trilinguisme des étudiants est à la fois une opportunité et un problème. II faut admettre qu'il présente un véritable défi aux enseignants de langues.

Nos principaux constats sur la question de l'acquisition du français- langue troisième, objet de notre étude sur le multilinguisme au cours des années 20072015, ont été publiés en 2007 (cf. aussi Widła, 2007, 2009, 2011, 2012, 2014, 2016). II résulte de cette étude que les apprenants s'appuient sur leurs différentes ressources langagières antérieures à l'apprentissage de cette nouvelle langue : 
Évaluation formative par le biais de pratiques « lernen durch lehren »...

Notre hypothèse voulant que les apprenants exploitent principalement les ressources des langues qu'ils jugent plus proches de la langue cible a été confirmée. La recherche a envisagé le type de matériel linguistique transféré : les sources des emprunts, le choix de la langue pour les règles morphosyntaxiques, les influences phonétiques. Les différentes tendances de choix de langue- « source d'inspiration » peuvent être expliquées aussi en termes de stades différents de l'appropriation de la L3 : les résultats montrent clairement qu'elles sont liées au niveau de compétence langagière des apprenants. Bien que la langue maternelle et la première langue étrangère jouent des rôles différents dans l'appropriation de la troisième langue, leur impact sur le degré de maîtrise de cette dernière varie en fonction du type de compétences acquises. De plus, il y a une corrélation positive des niveaux des compétences en $L 2$ et en L3. Les connaissances antérieures stimulent les progrès dans la plupart des cas analysés. De nombreux exemples viennent à l'appui de cette thèse. (Widła, 2018 : 57, cf. aussi Widła, 2007 : 199-201).

En prenant en considération le rôle de la biographie linguistique de l'apprenant trilingue (Widła 2007 : 152-154, cf. aussi Gabryś-Barker 2005), la question se pose de savoir comment diversifier l'offre didactique pour répondre à l'hétérogénéité des élèves. La première solution proposée par les didacticiens est l'individualisation de l'enseignement - dans le système traditionnel, elle est considérée comme extrêmement coûteuse; pourtant, à l'époque du multimédia, les nouvelles technologies permettent de personnaliser les approches en fonction du profil de l'élève. Les participants au séminaire en sont parfaitement conscients, c'est pourquoi de nombreux étudiants trilingues ont décidé de mettre en œuvre des projets destinés aux élèves trilingues qui se heurtent exactement aux mêmes difficultés linguistiques, en essayant d'enseigner comme ils auraient voulu qu'on leur enseigne quand eux-mêmes étaient débutants. Par exemple, les étudiants décident souvent de créer des modules qui améliorent la connaissance des structures et du vocabulaire en prenant en considération l'élimination d'interférences linguistiques (Debyser,1970 : 31-61). Ils savent comment profiter de leurs compétences de personnes trilingues pour raccourcir certaines étapes lorsque c'est possible, et accordent une importance particulière aux différences qui demandent le plus d'effort de la part des apprenants. Les exercices se basent donc généralement sur la comparaison, prêtant attention à un problème donné présenté dans deux langues (configuration L1-L3 ou L2-L3) ou trois (dans des triades : L1-L2-L3). Dans le cas de cours en ligne préparés par nos étudiants, les similitudes et les différences signalées concernent principalement le vocabulaire, mais de plus en plus d'étudiants abordent la question de la prononciation ou de l'orthographe, ainsi que la complexité de la grammaire. 


\section{Les feedbacks des étudiants reçus après les soutenances}

L'un des principes de l'évaluation de la notation est l'évaluation finale du travail de la part des étudiants. Ci-dessous, nous faisons le résumé de leurs retours. En réponse aux questions ouvertes sur la légitimité de la poursuite de la formule séminaire, les diplômés ont présenté, après leurs soutenances, les avantages suivants de cette approche:

- un vaste « terrain de jeu », une grande liberté dans le choix du sujet de la thèse de master,

- la possibilité pour l'étudiant d'acquérir des connaissances dans le domaine qui l'intéresse et de maîtriser parfaitement les questions non seulement théoriques, mais aussi linguistiques, notamment le vocabulaire et la grammaire,

- la possibilité pour l'étudiant d'utiliser sa propre créativité,

- un excellent soutien lors du travail sur le projet,

- la possibilité de travailler en français,

- la présentation des résultats de son travail devant un groupe,

- la possibilité de discussion à chaque étape de travail,

- les remarques de la part du responsable et des participants,

- la possibilité pour les étudiants d'être inspirés par les autres participants - en comparant leurs travaux respectifs sur la plateforme,

- une bonne ambiance pendant les études et pendant la soutenance,

- la possibilité offerte aux trilingues d'analyser les avantages et inconvénients de leurs compétences dans l'apprentissage d'une nouvelle langue afin de faire bénéficier les autres étudiants trilingues de leur expérience,

- vivre une aventure insolite dans ce travail si peu conventionnel,

- acquérir une bonne base de connaissances pour M oodle, permettant de maîtriser rapidement les fonctionnalités de la plateforme didactique,

- interactivité des cours en ligne,

- le séminaire, qui permet d'approfondir les connaissances sur les nouvelles technologies, non seulement dans le travail de l'enseignant, mais aussi pour les traducteurs, les employés de l'entreprise,

- avant tout, l'originalité (d'autres instituts proposent généralement le choix entre un séminaire de linguistique ou des études littéraires),

- adaptation aux exigences du marché du travail.

Voici l'exemple d'une réponse typique d'un diplômé à la question ouverte portant sur les avantages de l'approche proposée : 
Évaluation formative par le biais de pratiques « lernen durch lehren »...

« Libre choix du sujet de travail et de l'organisation du travail par l'étudiant - je ne le cache pas, c'est ce que j'ai le plus apprécié. II n'y avait pas de thèmes imposés, tout le monde travaillait lorsqu'il le pouvait (et je vous en remercie infiniment! Autrement, jamais de la vie, jamais je n'aurais réussi à achever le texte pendant l'année universitaire ...) chacun pouvait réaliser un projet qu'il souhaitait entreprendre. M on projet concernant le Jura cracovien était intéressant et difficile. Dans mon travail, j'ai dû recenser des écrits et de traduire beaucoup de matériaux. La littérature en français n'existait pas ... J'ai consacré beaucoup de temps à la traduction et à la gestion du projet informatique. Quant à l'exercice pratique en ligne - on ne peut le nier, dans le monde actuel, la compétence informatique est devenue la partie intégrante de la majorité des professions. Le travail sur Moodle m'a aidé à trouver un poste dans le secteur de transport et commerce (d'ailleurs, conforme, de plus, à mes habitudes : je peux parler à voIonté !). Eh bien, finalement je peux dire que je suis l'une des rares personnes capables de transmettre tant de savoir sur le Jura en français. »

Les diplômés restent dans un retour positif de cette expérience de séminaire ; cependant, nous croyons utile d'indiquer des limites à cette méthode d'apprentissage. Nous pensons surtout aux obstacles à la pensée créative liés aux émotions : la peur de l'échec, la réticence. Dans un cas pareil, une intervention pédagogique sera nécessaire pour affronter ces obstacles à l'intérieur du groupe. (cf. aussi Półtorak 2015).

\section{Conclusion}

La possibilité de gérer son apprentissage, ainsi que de celui des autres, est considérée par les futurs diplômés comme une préparation supplémentaire à la confrontation de leurs compétences à celles exigées par leurs futurs métiers. Ce passage de la connaissance à la compétence en langue et matière a été accompagné de la possibilité de créer une sorte de manuel en ligne, original et unique. L'apprentissage par enseignement auprès de futurs utilisateurs des cours d'une langue spécialisée exige la recherche d'idées originales, souvent fondées sur la communication réciproque entre les étudiants. (Martin, 2004, Półtorak 2011). L'intérêt porté dans le séminaire au processus d'apprentissage, chez les étudiants comme chez leurs élèves, devient une valeur ajoutée très enrichissante.

Compte tenu des possibilités actuelles de proposer une offre individuelle dans le domaine de la réception et de la production du langage écrit et parlé, ainsi que du désir de haute spécialisation dans des domaines précis ressenti par de nombreux étudiants de master en fonction de leurs besoins professionnels, il n'est pas surprenant que l'idée d'apprendre en enseignant ait gagné en popularité aux yeux de ceux-ci. On peut dire qu'il s'agit d'une variante de la forme 
classique de la méthode $L D L$, car dans le type de séminaire expérimenté, les étudiants ne contactent pas les utilisateurs de leurs cours. Cependant, ils créent ces derniers avec la conscience qu'ils serviront non seulement à l'auto-éducation, mais resteront également accessibles aux destinataires potentiels du réseau.

Le projet ne dispense pas de rédiger un mémoire de master classique ; les étudiants se basent sur un problème théorique, puis décrivent de manière extrêmement détaillée la mise en ligne des cours sur la plateforme, réalisée en toute autonomie.

Le travail systématique pendant toute la période de coopération permet un retour critique constructif depuis la planification de la méthode d'enseignement jusqu'à sa mise en œuvre ; à chaque étape, le responsable et tous les autres participants présentent leurs observations, aident à planifier, analyser et interpréter les problèmes conceptuels, didactiques, linguistiques et techniques.

Grâce aux bénéfices ressentis lors de l'évaluation formative, les étudiants de master se sentent bien préparés à l'épreuve finale. L'importance des compétences acquises dans un domaine recherché sur le marché de travail domine celle de réussir l'examen final, ce dernier ne devenant qu'une conséquence logique des efforts fournis. Le responsable, à son tour, n'a pas de souci à se faire quant à la motivation de l'étudiant.

\section{BIBLIOGRAPHIE}

Coyle D., Hood P., M arsh D. (2010), C.L.I.L. Content and Language Integrated Learning. Cambridge: CUP.

Debyser F. (1970), La linguistique contrastive et les interférences. „Langue française", n 8 (1070), 31-61.

Gabryś-Barker D. (2005), Aspects of multilingual storage, processing and retrieval. Katowice: Wydawnictwo Uniwersytetu Śląskiego.

Martin J-P. (1989), Quand les élèves font la classe. „Le Français dans le monde", $n^{\circ} 224,51-55$.

M artin J-P. (2004), Lernen durch Lehren : quand les apprenants font la classe. "Les Cahiers de l'APLIUT", nº 33 (1), 45-56.

PółtorakE. (2011), Question du feedback en didactique des langues étrangères : quelques réflexions théoriques. „Glottodidactica”, nXXXVIII, 31-41.

Półtorak E. (2015), Impact des nouvelles technologiessur les pratiques évaluatives en langues vivantes. Katowice : Wydawnictwo Uniwersytetu Śląskiego.

Widła H. (2007), L'acquisition du français - langue troisième. Problèmes méthodologiques et implications pratiques. Katowice: Wydawnictwo Uniwersytetu Śląskiego. 
Widła H. (2009), Ćwiczenia z języka francuskiego [eBook na płycie CD]. Dodatek do ksiqqżki: L'acquisition du français - langue troisième. Problèmes méthodologiques et implications pratiques. Katowice: Wydawnictwo Uniwersytetu Śląskiego.

Widła H. (2011), Skuteczność nauczania drugiego języka obcego - próba zastosowania wyników badań w praktyce. „Lingwistyka Stosowana”, n 4, 53-67.

Widła H. (2012), L'acquisition du français - langue troisième par le biais d'un environnement d'apprentissage en ligne, (in:) Berbinski S., Dobre D., Velicu A. (éds). Langage(s) et traduction. Bucuresti: Editura Universitatii din Bucuresti, 123-134.

Widła H., Półtorak E., Krajka J. (2014), E-learning in cultural studies instruction designing, implementing and evaluating a study programme, (in:) Smyrnova-Trybulska E. (éds). E-learning and Intercultural Competences Development In Different Countries. Katowice - Cieszyn: Studio NOA, 239-252.

Widła H. (2016), Znajomość Języków a znajomość kultur. "Języki Obce w Szkole”, $n^{\circ} 1,86-91$.

\section{SITOGRAPHIE}

http://el.us.edu.pl/demo/- plateforme contenant les cours réalisés dans le cadre du séminaire Multimedia [consulté 30.12.2018]

http://informatique.c0.pl/ cours de Grażyna Tkocz [consulté 29.12.2018]

https://apd.us.edu.pl/diplomas/100850/ mémoire de master de A-S. BastaSzczygieł [consulté20.11.2017]

https://apd.us.edu.pl/diplomas/106594/mémoire de master de M . Kurylak 2018

[consulté 14.12.2018]

https://apd.us.edu.pl/diplomas/108176/mémoire de master de N. Piwoń 2018

[consulté 20.12.2018]

https://apd.us.edu.pl/diplomas/108174/mémoire de master de M. M ajcher 2018 [consulté 19.12.2018] 